Series A

I. MATHEMATICA

414

\title{
ÜBER GANZE FUNKTIONEN MIT RATIONALEM MULTIPLIKATIONSTHEOREM
}

VON

P. J. MYRBERG

HEL I N K I 1968

S U O M A L A I EN TIEDEAKA TEM I A

doi:10.5186/aasfm.1968.414 
Vorgelegt am 8. März 1968

KESKUSKIRJAPAINO

HELSINKI 1968 


\section{Einleitung}

1. Nach Poincaré [9] besitzt die Funktionalgleichung

$$
\varphi(k z)=R(\varphi(z)),
$$

wo $R(y)$ eine gegebene rationale Funktion bezeichnet, als Lösung eine bis auf eine einfache Transformation bestimmte, in der ganzen komplexen $z$-Ebene meromorphe Funktion $\varphi(z, q)$, wenn $k=R^{\prime}(q)$, wo $q$ einen beliebigen repulsiven Fixpunkt der Abbildung

$$
y_{1}=R(y)
$$

bezeichnet. Die Funktion $\varphi$ reduziert sich auf eine ganze Funktion, wenn $R(y)$ ein Polynom ist. Es ist der Zweck der folgenden Untersuchungen, diesen speziellen Fall zu behandeln, obgleich gewisse von unseren Ergebnissen von der genannten Einschränkung unabhängig sind.

Die Untersuchung der Eigenschaften der Lösungen von $(1,1)$ wird in bekannter Weise auf das Problem der Iteration der rationalen Funktion $(1,2)$ zurückgeführt, deren allgemeine Theorie schon vor 50 Jahren von Fatou [1] und Julia [3] entwickelt worden ist. Es handelt sich hier um die Untersuchung der aus $R$ gemäss

$$
R_{n+1}=R_{n}(R)=R\left(R_{n}\right), \quad R_{1}=R
$$

erhaltenen unendlich vielen iterierten Funktionen $R_{n}$ und um die Bestimmung ihrer Grenzfunktionen in denjenigen Teilen der komplexen $z$-Ebene, wo sie eine normale Funktionenfamilie bilden. In allen bisher behandelten allgemeinen Fällen reduzieren sich die genannten Grenzfunktionen auf endlich viele Konstanten, welche dann Fixpunkte der Abbildung $(1,2)$ oder allgemeiner der Abbildung

$$
y_{n}=R_{n}(y)
$$

darstellen. Wir werden diesen Fall, auf den wir uns im folgenden beschränken werden, als normalen bezejchnen, indem die Existenz der anderen, singulären Fälle, bisher nur unter gewissen speziellen Umständen bestätigt worden ist.

Nach den vorbereitenden Betrachtungen von Kap. II, das eine zusammengestellte Darstellung von bisher schon bekannten Sätzen enthält, 
wird in den folgenden Kapiteln auf gewisse Eigenschaften der Lösungen von $(1,1)$ näher eingegangen. Insbesondere werden die asymptotischen Eigenschaften der betreffenden ganzen Funktionen untersucht, ferner wird die gegenseitige Abhängigkeit der zu den verschiedenen repulsiven Fixpunkten von $(1,2)$ gehörigen Lösungen von $(1,1)$ erklärt.

Als Anwendung wird zum Abschluss die allgemeine Lösung der Funktionalgleichung

$$
\varphi(k z)=a \varphi(z)+\varphi^{m}(z)=P(\varphi(z))
$$

bei reellem positivem Wert des Parameters a eingehend behandelt.

\section{Iteration der Polynome}

2. Es sei

$$
y_{1}=P(y)=y^{m}+a_{1} y^{m-1}+\ldots+a_{m}, \quad(m>1)
$$

ein beliebiges nichtlineares Polynom mit komplexen Koeffizienten, dessen erster Koeffizient gleich eins ist, was keine Einschränkung bedeutet. Durch $n$-fache Iteration erhält man aus $(2,1)$ ein Polynom mit dem Ausdruck

$$
y_{n}=P_{n}(y)=y^{m^{n}}+A_{1} y^{m^{n}-1}+\cdots+A^{m^{n}},
$$

wo die Koeffizienten ganze rationale Funktionen der Grössen $a_{v}$ mit nichtnegativen ganzen rationalen Koeffizienten sind. Die Fixpunkte der von $(2,1)$ vermittelten Abbildung, also die Wurzeln der Gleichung

$$
P(y)-y=0,
$$

werden in bekannter Weise, auf Grund der geometrischen Eigenschaften der Abbildung in der Umgebung des Fixpunktes, in attraktive, repulsive bzw. indifferente Fixpunkte eingeteilt, je nachdem ob für den Multiplikator $S=P^{\prime}(q)$

$$
|S|<1,>1 \text { oder }=1
$$

gilt.

Es sei nun $q$ ein Fixpunkt von $(2,2)$. Die aus $q$ durch Iteration von $(2,1)$ erhaltenen Bildpunkte

$$
q_{1}, q_{2}, q_{3}, \ldots
$$

die ebenfalls Fixpunkte von $(2,2)$ sind, werden bei der Iteration von $(2,1)$ miteinander zyklisch permutiert, wobei die Ordnung des Zyklus, d.h. die Anzahl der voneinander verschiedenen Punkte in der Folge $(2,3)$, gleich $n$ (oder gleich einem echten Teiler von $n$ ) ist. Weil der Multiplikator 


$$
P_{n}^{\prime}\left(q_{v}\right)=\prod_{\mu=1}^{n} P^{\prime}\left(q_{\mu}\right)
$$

für alle Punkte $(2,3)$ des Zyklus einen und denselben Wert besitzt, kann die obige Klassifizierung der Fixpunkte auf die Zyklen selbst übertragen werden.

Insbesondere für die attraktiven Zyklen gilt der fundamentale

Satz. Die Anzahl der attraktiven Zyklen von $(2,1)$ ist stets endlich und höchstens gleich $m-1$.

3. Bekanntlich gehört zu jedem attraktiven Fixpunkt $\alpha$ ein den Punkt enthaltendes Gebiet $D_{\alpha}$, dessen Punkte bei unbegrenzter Iteration der Abbildung gegen den Punkt $\alpha$ konvergieren. Dieses unmittelbare Attraktionsgebiet $D_{\alpha}$ von $\alpha$ ist stets entweder einfach oder unendlich vielfach zusammenhängend.

Wir betrachten nun die zu $(2,1)$ inverse, irrationale Funktion

$$
y_{-1}=P_{-1}(y)
$$

und ihre Iterierten

$$
y_{-v}=P_{-v}(y),
$$

welche alle in $D_{\alpha}$ vieldeutig sind, weil dort Verzweigungspunkte von $(3,1)$ stets vorhanden sind. Es sind hier zwei verschiedene Fälle möglich.

I. Fall. Mit $y$ gehören alle Punkte $P_{-1}(y)$ und somit auch alle Punkte $P_{-v}(y)$ zu $D_{\alpha}$. Man sagt, dass $D_{\alpha}$ dann vollständig invariant in bezug auf $(2,1)$ ist. In diesem Falle besteht $D_{\alpha}$ aus der Gesamtheit der Punkte der $y$-Ebene, deren Bildpunkte bei unbegrenzter Iteration von $(2,1)$ gegen den Punkt $\propto$ konvergieren.

II. Fall. Die Gebiete $P_{-1}\left(D_{\alpha}\right)$ fallen nicht sämtlich mit $D_{\alpha}$ zusammen. Die Gebiete

$$
P_{-r}\left(D_{\alpha}\right), \quad(v=1,2, \ldots)
$$

bilden dann eine unendliche Menge $\widetilde{D}_{\alpha}$ voneinander getrennt liegender Gebiete, die zusammen das vollständige (nicht zusammenhängende) Attraktionsgebiet von $\alpha$ ausmachen.

Wir bilden jetzt die Summe

$$
\sum_{v} \widetilde{D}_{\alpha_{v}}
$$

erstreckt über die Gesamtheit der Fixpunkte

$$
\alpha_{1}, \alpha_{2}, \alpha_{3}, \ldots
$$


aller bei $(2,1)$ vorhandenen attraktiven Zyklen. Aus den obigen Definitionen folgt, dass die Funktionen

$$
P_{n}(y), \quad(n=1,2,3, \ldots)
$$

in den Punkten von $(3,3)$ eine normale Familie bilden, deren Grenzfunktionen sich auf die Konstanten $(3,4)$ reduzieren. Es gilt somit

$$
\sum_{\nu} \widetilde{D}_{\alpha_{\nu}} \leqq D(P),
$$

wo $D(P)$ das Normalgebiet von $P$ bezeichnet, welches nach unserer Definition aus der Gesamtheit derjenigen Punkte der $y$-Ebene besteht, wo die Polynome $(3,5)$ eine normale Funktionenfamilie bilden. Die zu $D(P)$ komplementäre, abgeschlossene Menge $\Delta(P)$, also die Menge derjenigen Punkte, wo $(3,5)$ nicht normal ist, hat die Eigenschaft, dass jeder ihrer Punkte eine Häufungsstelle für die Punkte

$$
P_{-v}(y), \quad(v=1,2,3, \ldots)
$$

ist, wo $y$ einen beliebigen endlichen Punkt der komplexen $y$-Ebene, von einem einzigen höchstens abgesehen, bezeichnen kann.

Man hat hier zwischen zwei verschiedenen Fällen zu unterscheiden, je nachdem ob in $(3,6)$ das Zeichen $=$ oder $<$ gilt.

Im ersteren Falle reduzieren sich die Grenzfunktionen der normalen Funktionenfamilie $(3,5)$ auf die endlich vielen Konstanten $(3,4)$, die Fixpunkte der attraktiven Zyklen. Nach Fatou existiert dieser Fall sicher dann, wenn alle Verzweigungspunkte von $P_{-1}(y)$ zum Normalgebiet $D(P)$ gehören. Aus jedem Polynom der genannten Art kann man durch eine hinreichend kleine Variation der Koeffizienten neue Polynome ableiten, welche die nämlichen Eigenschaften fortwährend besitzen. Fatou [2] hat auch die bisher unbewiesene Vermutung ausgesprochen, der genannte Fall sollte als allgemeiner Fall angesehen werden, weil andere, singuläre Fälle wahrscheinlich nur dann auftreten können, wenn die Koeffizienten des Polynoms $(2,1)$ einer gewissen diskontinuierlichen Menge im betreffenden Koeffizientenraum angehören.

Jedenfalls kommt ein singulärer Fall vor, wenn unter den Grenzfunktionen auch nichtkonstante analytische Funktionen existieren und ferner, wenn die Anzahl der konstanten Grenzfunktionen unendlich ist.

Ein triviales Beispiel von einem singulären Fall mit nichtkonstanten Grenzfunktionen hat man in den linearen Polynomen

$$
P(y)=e^{i \omega} y+p, \quad \quad \omega / 2 \pi \text { irrational, }
$$

wo die Fixpunkte allerdings indifferent sind. Ein allgemeineres Beispiel für einen solchen Fall hat Sieger [10] beim Zentrumproblem gegeben. Beispiele 
von Polynomen mit unendlich vielen konstanten Grenzfunktionen liefern u.a. die von uns $[4,6]$ behandelten Binome

$$
P(y)=y^{m}+p
$$

bei singulärem Wert des Parameters $p$. Sie haben die bemerkenswerte Eigenschaft, dass man aus ihnen durch eine hinreichend kleine Variation des reellen Parameters $p$ Binome ableiten kann, die attraktive Zyklen beliebig hoher Ordnung besitzen.

4. Wir betrachten zum Abschluss den unendlich fernen Fixpunkt, der bei nichtlinearen Polynomen stets attraktiv ist.

Weil für alle $Z_{\text {weige }} P_{-1}(\infty)=\infty$ gilt, ist das zugehörige Attraktionsgebiet $D_{\infty}$ stets vollständig invariant. Nach der allgemeinen Theorie ist $D_{\infty}$ stets einfach zusammenhängend (Typus $A$ ) oder unendlich vielfach zusammenhängend. Im letzteren Falle besteht der Rand von $D_{\infty}$ entweder aus unendlich vielen Kontinuen (Typus B) oder aus einer unendlichen diskontinuierlichen Punktmenge (Typus $C$ ). Ein Kriterium für die Existenz eines bestimmten Typus ergibt sich aus dem Folgenden [1, S. 165].

Es seien

$$
\varrho_{1}, \varrho_{2}, \ldots, \varrho_{l}
$$

die Verzweigungspunkte von $P_{-1}(y)$. Man hat den Typus $A$, wenn keiner der Punkte $(4,1)$ zu $D_{\infty}$ gehört, den Typus $C$, wenn sie alle zu $D_{\infty}$ gehören. In den übrigen Fällen liegt der Typus $B$ vor.

Im Falle des Typus $A$ besteht der Rand $K$ von $D_{\infty}$ aus einem Kontinuum, das die Fixpunkte aller attraktiven Zyklen umschliesst. Als einfachsten Spezialfall (Untertypus $A_{0}$ ) kann derjenige angesehen werden, wo es einen einzigen endlichen attraktiven Fixpunkt $\alpha$ bei $(2,1)$ gibt, dessen Attraktionsgebiet $D_{\alpha}$ vollständig invariant ist. In diesem Falle ist $K$ eine Jordankurve, welche den gemeinsamen Rand von $D_{\alpha}$ und $D_{\infty}$ bildet. In den anderen Fällen enthält der Rand $K$ beim Typus $A$ unendlich viele Doppelpunkte.

Es sei wieder $\alpha$ ein attraktiver Fixpunkt von $(2,1)$ mit dem zugehörigen unmittelbaren Attraktionsgebiet $D_{\alpha}$. Bekanntlich enthält der Rand $K_{\alpha}$ von $D_{\alpha}$ wenigstens einen repulsiven Fixpunkt $q$, der gleichzeitig zum Rand von $D_{\infty}$ gehört.

Es sei ferner $\tilde{P}_{-1}(y)$ ein Zweig von $P_{-1}(y)$, welcher das Gebjet $D_{\alpha}$ auf sich selbst abbildet und gleichzeitig den Punkt $q$ invariant lässt. Ist dann $\lambda_{0}$ ein im Gebiet $D_{\alpha}$ verlaufender Kurvenbogen, dessen Endpunkte $a, b$ der Bedingung $b=P(a)$ genügen, so bilden die aus $\lambda_{0}$ durch Iteration von $P(y)$ bzw. $\widetilde{P}_{-1}(y)$ erhaltenen Bildbogen $\lambda_{n}, \lambda_{-n}$ zusammen eine bezüglich $P(y)$ und $\widetilde{P}_{-1}(y)$ invariante Linie 


$$
\lambda_{\alpha}=\sum_{n=-\infty}^{\infty} \lambda_{n}
$$

mit den Eigenschaften

$$
\lim _{n \rightarrow \infty} \lambda_{n}=\alpha, \lim _{n \rightarrow \infty} \lambda_{-n}=q .
$$

Die Linie $\lambda_{\alpha}$, welche eine endliche Länge besitzt, verbindet den attraktiven Fixpunkt $\alpha$ mit dem repulsiven Fixpunkt $q$, der somit für das Gebiet $D_{\alpha}$ wie auch für $D_{\infty}$ ein erreichbarer Punkt ist.

Man kann das obige Ergebnis noch verallgemeinern, indem man den attraktiven Fixpunkt $\alpha$ von $(2,1)$ durch die Fixpunkte

$$
\alpha_{1}, \alpha_{2}, \ldots, \alpha_{\mu}
$$

eines attraktiven Zyklus ersetzt, dessen zugehörige Gebiete einen repulsiven Fixpunkt $q$ von $(2,1)$ als gemeinsamen Randpunkt haben. Für jedes der genannten Gebjete ist $q$ gleichzeitig ein erreichbarer Punkt, welcher resp. mit den Punkten $(4,4)$ vermittels gewisser in den zugehörigen Gebieten verlaufenden Linien $\lambda_{\alpha_{\nu}}$ verbunden werden kann, die aus einander durch eine zyklische Permutation erhalten werden.

Wir bemerken zum Abschluss, dass beim Typus $C$ keine attraktiven Zyklen von endlichen Punkten existieren.

Beispiel. $y_{1}=y^{2}-p$. Bei reellem $p>-\frac{1}{4}$ ist $D_{\infty}$ von Typus $C$ für $p>2$ und sonst vom Typus $A$. Speziell für $p<\frac{3}{4}$ liegt der Untertypus $A_{0}$ vor [4].

Beispiele von Polynomen höheren Grades findet man in unseren Arbeiten [6] und [7].

\section{Einige Eigenschaften der $\varphi$-Funktionen}

\section{Die Nullstellen der Ableitung $\varphi^{\prime}(z)$.}

Es sei $q$ ein repulsiver Fixpunkt von $(2,1)$ und $q(z)=\varphi(z, q)$ eine zugehörige ganze Lösung der Gleichung

$$
\varphi(k z)=P(\varphi(z)),
$$

wo

$$
\varphi(0)=q, \quad k=P^{\prime}(q), \quad \varphi^{\prime}(0) \neq 0 .
$$

Aus der durch Differentiation erhaltenen Gleichung

$$
k \varphi^{\prime}(k z)=P^{\prime}(\varphi(z)) \varphi^{\prime}(z)
$$

geht hervor, dass mit $z_{0}$ zugleich alle Punkte 


$$
z_{v}=k^{v} z_{0}, \quad(v=1,2, \ldots)
$$

Nullstellen von $\varphi^{\prime}(z)$ sind.

Aus $(5,2)$ folgt umgekehrt

$$
\varphi^{\prime}(z)=\frac{1}{k} P^{\prime}\left(\varphi\left(\frac{z}{k}\right)\right) \varphi^{\prime}\left(\frac{z}{k}\right)
$$

und hieraus für $\varphi^{\prime}\left(z_{0}\right)=0$,

$$
\text { entweder } \quad \varphi^{\prime}\left(\frac{z_{0}}{k}\right)=0 \text { oder } P^{\prime}\left(\varphi\left(\frac{z_{0}}{k}\right)\right)=0 \text {. }
$$

Im ersteren Falle gilt

$$
\text { entweder } \quad \varphi^{\prime}\left(\frac{z_{0}}{k^{2}}\right)=0 \quad \text { oder } \quad P^{\prime}\left(\varphi\left(\frac{z_{0}}{k^{2}}\right)\right)=0
$$

usw. Wegen $\varphi^{\prime}(0) \neq 0$ muss schliesslich für eine Zahl $n$ gelten

$$
\varphi^{\prime}\left(\frac{z_{0}}{k^{n}}\right) \neq 0, P^{\prime}\left(\varphi\left(\frac{z_{0}}{k^{n}}\right)\right)=0 .
$$

Wir setzen $\frac{z_{0}}{k^{n}}=z_{\mu}$ und gelangen zum

Satz 1. Man bekommt alle Nullstellen von $\varphi^{\prime}(z)$ aus

$$
z_{v u}=k^{v} z_{u} \quad(v=1,2, \ldots),
$$

wo $z_{\mu}$ die Gesamtheit der $z$-Werte zu durchlaufen hat, für welche die Gleichungen

$$
\varphi\left(z_{\mu}\right)=x_{\mu}, \quad P^{\prime}\left(x_{\mu}\right)=0
$$

gelten. Die Punkte $(5,4)$ liegen bei reellem $k^{n}$ auf gewissen vom Nullpunkt ausgehenden Strahlen, sonst auf logarithmischen Spiralen.

Eine Ausnahme bildet derjenige spezielle Fall, wo $P^{\prime}(y)$ eine einzige Nullstelle $y_{0}$ besitzt, die zugleich ein Picardscher Ausnahmewert von $\varphi(z)$ ist. Dann hat $P(y)$ den Ausdruck

$$
P(y)=a+b\left(y-y_{0}\right)^{m},
$$

und die zugehörige $\varphi$-Funktion reduziert sich auf eine Exponentialfunktion.

6. Die inverse Funktion

$$
z=\psi(y)
$$


von $\varphi(z)$ ist eine unendlich vieldeutige Funktion, deren zugehörige Riemannsche Fläche algebraische Verzweigungspunkte in den Bildpunkten

$$
p_{\mu, \nu}=\varphi\left(z_{\mu, v}\right)
$$

der Nullstellen von $\varphi^{\prime}(z)$ besitzt. Für den durch die Bedingung $\tilde{\psi}(q)=0$ eindeutig bestimmten Hauptzweig von (6,1), welcher der Schröderschen Funktionalgleichung

$$
\tilde{\psi}(P(y))=k \tilde{\psi}(y)
$$

genügt, gilt nach PICARD [8] die Darstellung

$$
\tilde{\psi}(y)=\lim _{n \rightarrow \infty} k^{n}\left[\bar{P}_{-n}(y)-q\right] .
$$

Beispiele. Bei der Funktion $\varphi=e^{z}$ mit der Funktionalgleichung

$$
\varphi(2 z)=\varphi^{2}(z)
$$

ergibt sich aus (6,3) für die inverse Funktion, den Hauptzweig des Logarithmus $\overline{\log } y$, die Darstellung

$$
\overline{\log } y=\lim _{n \rightarrow \infty} 2^{n}\left[y^{1 / 2^{n}}-1\right] .
$$

Es sei zweitens $P(y)=y^{2}-p$, wo $p$ reell und $>-\frac{1}{4}$. Wegen $\bar{P}_{-1}(y)$ $=\sqrt{p+y}$ ergibt sich aus $(6,3)$ die Darstellung

$$
\tilde{\psi}(y)=\lim _{n \rightarrow \infty} k^{n}[\sqrt{p+\sqrt{p+\cdots+\sqrt{p+y}}}-q]
$$

für die inverse Funktion der zum repulsiven Fixpunkt $q=\frac{1}{2}+\sqrt{\frac{1}{4}+p}$ gehörigen Lösung der Funktionalgleichung

$$
\varphi(k z)=\varphi^{2}(z)-p, \text { wo } k=2 q .
$$

\section{Die asymptotischen Werte von $\phi(x, q)$.}

Es sei wieder $\varphi(z, q)$ die zum repulsiven Fixpunkt $q$ von $(2,1)$ gehörige $\varphi$-Funktion und $z=\widetilde{\psi}(y)$ der Hauptzweig ihrer inversen Funktion. Durch $\tilde{\psi}(y)$ wird die Umgebung des Punktes $y=q$ konform auf eine Umgebung des Nullpunktes $z=0$ derart, abgebildet dass die Abbildung $y_{1}=P(y)$ in die umkehrbar eindeutige, lineare Abbildung

$$
z_{1}=k z
$$

transformiert wird.

Es sei nun $(4,4)$ ein Zyklus attraktiver Punkte, deren Gebiete $D_{\alpha_{v}}$ den Punkt $q$ als gemeinsamen Randpunkt haben. Den dort liegenden Linien 
$\lambda_{\alpha_{v}}$ entsprechen in der $z$-Ebene gewisse vom Nullpunkt ausgehende unendliche Linien $l_{v}$, die von $(7,1)$ miteinander zyklisch permutiert werden und auf denen die Funktion $\varphi(z, q)$ die asymptotischen Werte $(4,4)$ besitzt. Die genannten Linien haben im allgemeinen die Form einer logarithmischen Spirale.

Man bekommt eine Abschätzung für die Anzahl der asymptotischen Werte von $\varphi$, wenn man von dem nach $(2,2)$ geltenden Ausdruck

$$
\varphi\left(k^{n} z\right)=P_{n}(\varphi(z))=(\varphi(z))^{m^{n}}+\cdots
$$

ausgeht, woraus für $|z|=1,\left|k^{n}\right|=r$ eine Ungleichung von der Form

$$
\left|\varphi\left(k^{n} z\right)\right|<C^{m^{n}}
$$

erhalten wird, wo $C$ eine endliche Konstante bezeichnet. Wegen

$$
m^{n}=r^{b}, \quad b=\frac{\log m}{\log |k|}
$$

folgt hieraus für $|z|=r$

$$
|\log \varphi(z)| \leqq r^{b} \log C .
$$

Aus dem bekannten Satz von Denjoy-Ahlfors folgt hieraus der

Satz 2. Für die Anzahl $N$ der endlichen asymptotischen Werte von $\varphi(z, q)$ gilt die Abschätzung

$$
N \leqq 2 b \leqq \frac{2 \log m}{\log |k|}
$$

Es sei nun $L$ ein unendlicher Weg, wo $\varphi$ den asymptotischen Wert $\alpha$ hat. Dann hat $\varphi$ auf dem Weg $k^{v} L$ den asymptotischen Wert $P_{v}(\alpha)$. Weil es nun nach dem obigen Satz nur endlich viele asymptotische Werte gibt, muss für einen Wert $n$

$$
P_{n}(\alpha)=\alpha
$$

gelten. Wir haben somit den

Satz 3. Die asymptotischen Werte von $\varphi$ bestehen aus den Fixpunkten von gewissen Zyklen von $(2,1)$.

Diese Zyklen sind wenigstens bei den normalen Polynomen attraktiv.

Zur Begründung des zweiten Teiles unseres Satzes bemerken wir, dass bei den normalen Polynomen die Verzweigungspunkte von $\psi(y)$, welche mit den Bildpunkten der Verzweigungspunkte von $P_{-1}(y)$ zusammenfallen, innerhalb des Normalgebietes des Polynoms liegen, während die repulsiven (und indifferenten) Fixpunkte stets zum Rand des Gebietes gehören. Die Bildpunkte der repulsiven Fixpunkte in der $z$-Ebene liegen daher im 
Endlichen, und sie können deshalb keine asymptotischen Werte von $\varphi$ darstellen.

Beispiel. $P(y)=y^{2}-p, p=$ reell $>-\frac{1}{4}$. Weil $k=2 q$ für den repulsiven Fixpunkt $q=\frac{1}{2}+\sqrt{\frac{1}{4}+p}$ ist, so ergibt sich aus $(7,3)$ $N \leqq 1$ für $p>0$. Tatsächlich ist $N=1$ nur für $p<\frac{3}{4}$, sonst ist $N=0$. Für $p \leqq 0$ hat die Abschätzung $(7,3)$ keine praktische Bedeutung.

\section{Gegenseitige Abhängigkeit der verschiedenen $\varphi$-Funktionen.}

Es sei

$$
q_{1}, q_{2}, \ldots, q_{n}
$$

ein repulsiver Zyklus von $(2,1)$. Für die zugehörigen $\varphi$-Funktionen

$$
\varphi_{v}(z)=\varphi\left(z, q_{v}\right)
$$

also für die Lösungen von

gilt der

$$
\varphi_{\nu}(k z)=P_{n}\left(\varphi_{\nu}(z)\right), \text { wo } k=\prod_{\nu=1}^{n} P^{\prime}\left(q_{v}\right)
$$

Satz 4. Die Funktionen $(8,2)$ werden aus einander zyklisch aus

$$
\varphi_{\nu+1}(z)=P\left(\varphi_{\nu}(z)\right)
$$

erhalten.

Beweis. Aus

$$
\varphi_{1}(k z)=P_{n}\left(\varphi_{1}(z)\right), \quad \varphi_{1}(0)=q_{1}
$$

ergibt sich für die Funktion $\varphi_{2}(z)=P\left(\varphi_{1}(z)\right)$

$\varphi_{2}(k z)=P\left(\varphi_{1}(k z)\right)=P\left(P_{n}\left(\varphi_{1}(z)\right)\right)=P_{n}\left(P\left(\varphi_{1}(z)\right)\right)=P_{n}\left(\varphi_{2}(z)\right), \varphi_{2}(0)=q_{2}$,

usw. In analoger Weise kann man den folgenden etwas allgemeineren Satz beweisen.

Satz 5. Es sei $S$ ein mit $P$ vertauschbares Polynom, also $P S=S P$. Ist dann $\varphi_{1}(z)$ eine zum repulsiven Fixpunkt $q_{1}$ von $(2,1)$ gehörige Lösung von

so gibt

$$
\varphi_{1}(k z)=P\left(\varphi_{1}(z)\right)
$$

$$
\varphi_{2}(z)=S\left(\varphi_{1}(z)\right)
$$

eine zum Fixpunkt $q_{2}=S\left(q_{1}\right)$ gehörige Lösung.

Beispiel

$$
P(y)=a y+y^{m}, \quad S(y)=\varepsilon y .
$$

Wegen

$$
P S=a \varepsilon y+\varepsilon y^{m}, \quad S P=a \varepsilon y+\varepsilon^{m} y^{m}
$$


gilt $P S=S P$, wenn $\varepsilon^{m-1}=1$. Ist somit $\varphi(z)$ eine Lösung der Gleichung

$$
\varphi(k z)=a \varphi(z)+\varphi^{m}(z),
$$

so hat diese Gleichung gleichzeitig die Lösungen

$$
e^{\frac{2 \pi i v}{m-1}} \varphi(z), \quad(v=1,2, \ldots, m-2) .
$$

IV. Die Funktionalgleichung $\varphi(k z)=a \varphi(z)+\varphi^{m}(z)$.

9. Es handelt sich im folgenden zuerst um die Untersuchung der Iteration des Polynoms

$$
P(y)=a y+y^{m}, \quad(m \geqq 2)
$$

wo der Parameter a reell und positiv angenommen wird. Im einfachsten Falle $m=2$ kann das Polynom durch die Substitution $y=x-\frac{a}{2}$ in das Polynom

$$
x_{1}=x^{2}-p, \text { wo } p=\frac{a^{2}}{4}-\frac{a}{2}
$$

transformiert werden, welches wir schon früher [4] ausführlich behandelt haben.

10. Typus von $D_{\infty}$.

Aus der durch Differentiation erhaltenen Gleichung

$$
P^{\prime}(y)=a+m y^{m-1}=0
$$

bekommt man für die Verzweigungspunkte der inversen Abbildung $P_{-1}(y)$ den Ausdruck

$$
\varrho_{\nu}=\varrho \varepsilon_{m-1}^{v}, \quad(v=0,1,2, \ldots, m-2)
$$

wo $\varepsilon_{m-1}=e^{\frac{2 \pi i}{m-1}}$ und

$$
\varrho_{0}=\varrho=a \frac{m-1}{m}\left(-\frac{a}{m}\right)^{1 / m-1} .
$$

Der aus $(10,1)$ durch $n$-fache Iteration erhaltene Bildpunkt hat offenbar einen Ausdruck von der Form

$$
\varrho_{v}^{(n)}=b_{n} \varrho_{v},
$$

wo $b_{n}$ eine von $v$ unabhängige reelle Grösse bezeichnet. 
Es sei $m$ zuerst eine gerade Zahl, in welchem Falle $\varrho$ reell und negativ ist. Aus

$$
\varrho^{(1)}=P(\varrho)=a \varrho\left[1-\frac{(m-1)^{m-1} a^{m-1}}{m^{m}}\right]
$$

geht hervor, dass für

$$
a \leqq \frac{m^{m / m-1}}{m-1}=m_{2}
$$

$\varrho^{(1)} \leqq 0$ ist. Es ist $P(y)=0$ für $y=0$ und für $y=-\sqrt[m-1]{a}=b$. Ferner ist das Minimum von $P(y)$ gleich $\varrho$, das in einem Punkt des Intervalls $(0, b)$ erreicht wird.

Weil $b \leqq P(y) \leqq 0$ für $b \leqq y \leqq 0$, so gilt allgemein $\left|\varrho^{(n)}\right|<b$ und der Typus von $D_{\infty}$ ist $A$.

Es sei nachher $a>m_{2}$. Dann ist $\varrho^{(1)}>0$, und die Grössen $\varrho^{(n)}$ bilden eine monoton wachsende Folge von positiven Grössen. Der Grenzwert $\lim _{n \rightarrow \infty} \varrho^{(n)}$, der offenbar einen Fixpunkt von $(9,1)$ liefert, kann nur unendlich sein. Der Typus von $D_{\infty}$ ist jetzt $C$.

Es sei zweitens $m$ eine ungerade Zahl, in welchem Falle alle Punkte $\varrho_{v}$ imaginär sind. Wir setzen $y=\varrho t$, wo $t$ reell ist, und erhalten für das transformierte Polynom aus $(9,1)$ den Ausdruck

$$
Q(t)=t\left(a+\varrho^{m-1} t^{m-1}\right),
$$

wo

$$
\varrho^{m-1}=-\frac{a^{m}}{m^{m}}(m-1)^{m-1}
$$

Das Polynom $Q(t)$ hat als reelle Nullpunkte die Punkte

$$
t=0, t= \pm \frac{m_{2}}{a}
$$

und sein Maximum $t_{1}=+1$ bzw. Minimum $t_{1}=-1$ wird in einem Punkt des Intervalls $\left(0, \frac{m_{2}}{a}\right)$ bzw. $\left(0,-\frac{m_{2}}{a}\right)$ erreicht.

Wir bezeichnen nun mit $d$ die positive Wurzel der Gleichung

$$
Q(t)=-1
$$

und betrachten zuerst den

Fall $d=1$. Aus $a+\varrho^{m-1}=-1$ und $(10,6)$ erhält man zur Bestimmung von a die Gleichung 


$$
a^{m}=m_{2}^{m-1}(a+1),
$$

welche die negative reelle Doppelwurzel $a=-\frac{m}{m-1}$ besitzt. Die an diesem Punkt gezogene Tangente der Kurve $y=a^{m}$ schneidet diese Kurve noch in einem Punkt $a=m_{3}>m_{2}$, welcher die einzige reelle positive Wurzel der Gleichung $(10,7)$ liefert. Weil für $a=m_{3}$ die Grössen $t, t_{1}, t_{2}, \ldots$ alternierend gleich -1 und +1 sind, so ist für $a=m_{3}$ das Gebiet $D_{\infty}$ vom Typus $A$.

$\operatorname{Im}$ Falle $d>1$ gilt für $|t|<d$ allgemein $\left|t_{n}\right|<d$, und $D_{\infty}$ ist vom Typus $A$.

Schliesslich ist im Falle $d<1$ für $t=1$

$$
\left|t_{1}\right|<\left|t_{2}\right|<\ldots
$$

und $\lim t_{n}=\infty$, der Typus somit $C$.

$n \rightarrow \infty$

Zusammenfassend bekommt man also den

Satz 6. Der Typus von $D_{\infty}$ ist $A$ für $a \leqq \omega_{m}$ und $C$ für $a>\omega_{m}$. Hier ist bei geradem $m$

$$
\omega_{m}=m_{2}=\frac{m^{m / m-1}}{m-1}
$$

und bei ungeradem $m$

$$
\omega_{m}=m_{3}>m_{2},
$$

wo $m_{3}$ die einzige positive reelle Wurzel der Gleichung

$$
a^{m}=m_{2}^{m-1}(a+1)
$$

bezeichnet.

Zum Beispiel für $m=2$ ist $m_{2}=4$ und für $m=3$ ist

$$
m_{2}=\frac{3}{2} \sqrt{3}, m_{3}=3 .
$$

11. Bestimmung der attraktiven Fixpunkte der Abbildung $(9,1)$.

Aus der Gleichung

$$
P(y)=a y+y^{m}=y
$$

bekommt man als Lösungen neben $y=0$ die gesuchten Fixpunkte

$$
q_{v}=(1-a)^{1 / m-1} \varepsilon_{m-1}^{v}, \quad(v=0,1,2, \ldots, m-2) .
$$

Sie liegen auf dem Kreis $|y|=\left|q_{0}\right|$ und gehören zu den Strahlen

$$
\arg y=\frac{2 v+1}{m-1} \pi=\omega_{v},
$$


deren zugehörige Geraden bezüglich $(9,1)$ invariant sind. Diese Invarianteneigenschaft haben auch die dazwischen liegenden Strahlen

$$
\arg y=\frac{2 v}{m-1} \pi=\tilde{\omega}_{v} .
$$

Aus dem Ausdruck

$$
S_{v}=a+m q_{v}^{m-1}=a+m(1-a)
$$

des zum Fixpunkt $q_{v}$ gehörigen Multiplikators geht hervor, dass die Punkte $(11,1)$ gleichzeitig attraktiv für

$$
1<a<m_{1}=\frac{m+1}{m-1}
$$

sind, sonst repulsiv oder indifferent. Speziell für $a_{0}=\frac{m}{m-1}$ ist der Multiplikator gleich null.

Der Fixpunkt $y=0$ ist attraktiv für $a<1$, repulsiv für $a>1$ und indifferent für $a=1$.

\section{Attraktive Zyklen höherer Ordnung.}

Nach der allgemeinen Theorie können attraktive Zyklen zweiter oder höherer Ordnung nur für die Parameterwerte

$$
m_{1}<a<\omega_{m}
$$

existieren, und ihre Anzahl ist nach dem Satz von Nr. 2 höchstens $m-1$.

Wir betrachten im folgenden etwas näher die Zyklen zweiter Ordnung, deren Fixpunkte als Fixpunkte der Abbildung $y_{2}=P_{2}(y)$ der Gleichung

$$
a\left(a y+y^{m}\right)+\left(a y+y^{m}\right)^{m}=y
$$

genügen. Nach Fortlassen des Faktors $y$ und durch Einsetzung

$$
y^{m-1}=t-a
$$

gelangt man zur Gleichung

$$
g(t)=t^{m+1}-a t^{m}+a t-1=0,
$$

deren triviale Wurzel $t=1$ die Fixpunkte von $(9,1)$ liefert. Elementare Betrachtungen zeigen, dass von den übrigen Wurzeln ron $(12,3)$ stets zwei, $t_{1}, t_{2}$, reell und positiv sind mit den Eigenschaften

$$
0<t_{1}<1<t_{2}<a .
$$

Bei ungeradem $m$ hat $(12,3)$ noch die negative reelle Wurzel $t=-1$, welche stets zu einem repulsiven Zyklus führt. 
Zur Bestimmung der attraktiven Zyklen gehen wir von dem Gleichungspaar

$$
y_{2}=y, y_{2}^{\prime}=1
$$

aus, welches die indifferenten Zyklen mit dem Multiplikator $S=+1$ liefert. Das Gleichungspaar $(12,4)$ ist, wie leicht einzusehen, äquivalent mit

$$
g(t)=0, \quad g^{\prime}(t)=1,
$$

welches nur die einzige Lösung $t=1$ besitzt.

Ferner ergibt sich aus

$$
y_{2}^{\prime}=\left(a+m y^{m-1}\right)\left(a+m y_{1}^{m-1}\right)=0
$$

zur Bestimmung der Nullzyklen für $a$ die Gleichung

$$
a^{m+1}=m_{2}^{m-1}\left(a^{2}-a_{0}\right),
$$

welche neben $a=a_{0}$ eine einzige reelle und positive Lösung besitzt für die $a$-Werte des Intervalls

$$
m_{1}<a<m_{-1}<m_{2},
$$

wo für $a=m_{-1}$ ein indifferenter Zyklus mit dem Multiplikator $S=-1$ vorkommt. Wegen der stetigen Abhängigkeit des Multiplikators $S$ vom Parameter $a$ ergibt sich aus dem Obigen der

Satz 7. Die Abbildung $(9,1)$ besitzt attraktive Zyklen zweiter Ordnung sicher für die Parameterwerte $(12,6)$.

Die Anzahl solcher Zyklen ist genau $m-1$, und ihre Fixpunkte

$$
q_{v}^{\prime}=q_{0}^{\prime} \varepsilon_{m-1}^{v}, \quad q_{v}^{\prime \prime}=q_{0}^{\prime \prime} \varepsilon_{m-1}^{v}
$$

sind auf den Strahlen $(11,2)$ so gelegen, dass zwischen ihnen stets ein repulsiver Fixpunkt $q_{v}$ von $(9,1)$ vorhanden ist.

13. Als Beispiel betrachten wir den

Fall $m=3$. Jetzt lautet die Gleichung $(12,3)$

$$
t^{4}-a t^{3}+a t-1=0,
$$

welche neben $t= \pm 1$ die positiven reellen Wurzeln

$$
t=\frac{a}{2} \pm \sqrt{\frac{a^{2}}{4}-1}=\alpha \pm
$$

besitzt, denen die rein imaginären $y$-Werte

$$
\eta_{2}^{ \pm}= \pm i \sqrt{\alpha^{-}}, \eta_{3}^{+}= \pm i \sqrt{\alpha^{+}}
$$


entsprechen. Die den Werten $t=\mp 1$ entsprechenden $y$-Werte

$$
\eta_{1}^{ \pm}= \pm i \sqrt{a+1}, \quad \eta_{0}^{ \pm}= \pm i \sqrt{a-1}
$$

sind ebenfalls imaginär.

Man hat hier drei Zyklen zweiter Ordnung

$$
\Sigma_{1}\left(\eta_{1},-\eta_{1}\right), \quad \Sigma_{2}\left(\eta_{2}, \eta_{3}\right), \quad \Sigma_{3}\left(-\eta_{2},-\eta_{3}\right)
$$

resp. mit den Multiplikatoren

$$
S_{1}=(2 a+3)^{2}, \quad S_{2}=S_{3}=9-2 a^{2} .
$$

Hieraus geht hervor, dass $\Sigma_{1}$ stets repulsiv ist, während $\Sigma_{2}$ und $\Sigma_{3}$ attraktiv sind für die $a$-Werte des Intervalls

$$
2<a<\sqrt{5}
$$

welches mit $(12,6)$ identisch ist.

14. Iteration von $(9,1)$. Es sei zuerst $a<1$, in welchem Falle der Nullpunkt $y=0$ der einzige endliche attraktive Fixpunkt von $(9,1)$ ist. Man kann zeigen, dass das zugehörige vollständig invariante Attraktionsgebiet aus dem Inneren einer geschlossenen Jordankurve $K$ besteht, auf welcher sämtliche repulsiven Fixpunkte $(9,1)$ gelegen sind. (Vgl. Fatou [1, S. 100], wo der Fall $a=\frac{1}{2}$ eingehend behandelt worden ist.)

Es gehöre zweitens der Parameter a zum Intervall $(11,4)$, in welchem Falle die Fixpunkte $q_{v}$ attraktiv sind. Elementare Betrachtungen zeigen, dass jetzt auf den Strahlen $(11,2)$ die Gleichung

$$
\lim _{n \rightarrow \infty} y_{n}=q_{v}
$$

für die Punkte einer vom Nullpunkt ausgehenden und den Punkt $q_{v}$ enthaltenden Strecke $\tau_{\nu}$ gültig ist, während auf den Strahlen $(11,3)$

$$
\lim _{n \rightarrow \infty} y_{n}=\infty
$$

für $y \neq 0$ gilt. Es gehört somit die Strecke $\tau_{\nu}$ als ganzes zum unmittelbaren Attraktionsgebiet $D_{q_{v}}$ von $q_{v}$, während die Strahlen $(11,3)$ als ganzes zum Gebiet $D_{\infty}$ gehören. Dabei ist der Nullpunkt ein gemeinsamer Randpunkt für alle genannten Gebiete.

Es gehöre drittens a zum Intervall $(12,6)$.

Für jeden Strahl $(11,2)$ ist der dort liegende Fixpunkt $q_{v}$ von $(9,1)$ jetzt repulsiv und gemeinsamer Endpunkt für zwei Strecken $\tau_{v}^{\prime}, \tau_{v}^{\prime \prime}$ von $(11,2)$, welche den attraktiven Fixpunkt $q_{v}^{\prime}$ bzw. $q_{v}^{\prime \prime}$ von $P_{2}(y)$ als inneren Punkt haben. 
Auf den Strecken $\tau_{v}^{\prime}, \tau_{v}^{\prime \prime}$, welche von $(9,1)$ miteinander vertauscht werden, gilt bzw.

$$
\lim _{n \rightarrow \infty} y_{2 n}=q_{\nu}^{\prime}, \quad \lim _{n \rightarrow \infty} y_{2 n}=q_{\nu}^{\prime \prime} .
$$

Die genannten Strecken gehören als Ganzes zum Attraktionsgebiet $D_{q_{\nu}}^{\prime}$ bzw. $D_{q_{v}}^{\prime \prime}$ bei Iteration von $P_{2}(y)$.

Übrig ist noch der Fall

$$
m_{-1}<a<\omega_{m}
$$

wo bei $(9,1)$ attraktive Zyklen beliebig hoher Ordnung existieren können. Wir können hier allerdings auf diesen Fall, wo schon für $m=2$ Erscheinungen sehr komplizierter Natur vorkommen, nicht näher eingehen [4].

15. Die asymptotischen Werte von $\varphi(z, q)$.

Wir betrachten zuerst den Fall $q=0$, wo $k=a>1$. Wird die zugehörige Funktion $\varphi(z)=\varphi(z, 0)$ gemäss $\varphi^{\prime}(0)=1$ normiert, so bekommt man eine für die reellen $z$-Werte reelle ganze Funktion mit den folgenden Eigenschaften.

Die Funktion $\varphi(z, 0)$ hat auf den Strahlen

$$
\arg z=\frac{2 v+1}{m-1} \pi=\omega_{v}
$$

den asymptotischen Wert $q_{v}$, wenn $1<a<m_{1}$. Auf den Strahlen $\arg z=\tilde{\omega}_{v}$ gilt jedenfalls für $a>1$

$$
\lim _{z \rightarrow \infty} \varphi(z, 0)=\infty \text {. }
$$

Die zum Fixpunkt $q_{v}$ gehörige Lösung $\varphi_{v}(z)=\varphi\left(z, q_{v}\right)$ existiert nach Nr. 12 für

$$
m_{1}<a<m_{-1} \text { und für } a<1 .
$$

In den beiden Fällen ist die aus $\varphi_{v}(z)$ abgeleitete Funktion

$$
\psi_{v}(t)=e^{-i \omega_{\nu}} \varphi_{v}\left(t e^{i \omega_{v}}\right)
$$

eine für reelle $t$-Werte reelle Funktion. Sie genügt der Gleichung

$$
\psi_{\nu}(k t)=a \psi_{\nu}(t)-\psi_{\nu}^{m}(t),
$$

und sie besitzt für reelle $t$ die Grenzwerte

$$
\lim _{t \rightarrow-\infty} \psi_{\nu}(t)=e^{-i \omega_{\nu}} q_{\nu}^{\prime}, \lim _{t \rightarrow \infty} \psi_{\nu}(t)=e^{-i \omega_{\nu}} q_{\nu}^{\prime \prime} .
$$

Dies gilt auch für $a<1$, wo $q_{v}^{\prime}=0, q_{v}^{\prime \prime}=\infty$ zu setzen ist. 
Aus $(15,3)$ folgt nachher, dass die Funktion $\varphi_{\nu}(z)$ auf den Strahlen $\arg z= \pm \omega_{v}$ resp. die asymptotischen Werte $q_{v}^{\prime}$ und $q_{v}^{\prime \prime}$ besitzt. Auf den Strahlen $\arg z= \pm \tilde{\omega}_{\nu}$ gilt fortwährend $(15,2)$.

Was den oben ausgeschlossenen Fall $a>m_{-1}$ betrifft, können wir hier nur folgendes bemerken.

Weil für $a>\omega_{m}$ keine attraktiven Zyklen existieren, hat dann $\varphi(z)$ keinen endlichen asymptotischen Wert. Dies gilt wenigstens im Falle $m=2$ auch für die Werte $(\mathbf{1 4}, 3)$ des Parameters, wo zwar attraktive Zyklen beliebig hoher Ordnung existieren können, die zugehörigen Attraktionsgebiete $D$ aber die Fixpunkte $q_{v}, 0$ niemals als Randpunkte besitzen. Bei solchen Parameterwerten bekommt man jedenfalls ganze Funktionen mit endlichen asymptotischen Werten, wenn die Funktionalgleichung $(1,5)$ durch eine allgemeinere von der Form

ersetzt wird.

$$
\varphi(k z)=P_{n}(\varphi(z))
$$

\section{Literatur}

[1] Fatou, P.: Sur les équations fonctionnelles. - Bull. Soc. Math. de France 47 (1919) et 48 (1920).

[2] - - Sur les frontières de certains domaines. - Ibidem 51 (1923).

[3] Julia, G.: Mémoire sur l'itération des fractions rationnelles. - J. Journal de Math., 7 série, t. 4 (1918).

[4] Myrberg, P. J.: Iteration der reellen Polynome zweiten Grades. - I. Ann. Acad. Sci. Fennịcæ 256 (1958). II. Ibidem 268 (1959). III. Ibidem 336 (1964).

[5] - -- Sur l'itération des polynomes réels quadratiques. - Journal de Math., 4, t. 41 (1962).

[6] - - Iteration der Binome beliebigen Grades. - Ann. Acad. Sci. Fennicæ 348 (1964).

[7] -》- Iteration der Polynome mit reellen Koeffizienten. - Ibidem 374 (1965).

[8] Picard, E.: Leçons sur quelques équations fonctionnelles. - Paris 1928.

[9] Porncaré, H.: Sur une classe nouvelle de transcendantes uniformes. - Journ. de Liouville, 4 série, t. 6 (1890).

[10] Siegel, C. L.: Iteration of analytic functions. - Ann. of Math. 43 (1942). 